\title{
PROMOVIENDO LA COMPETENCIA SOCIOEMOCIONAL EN LA INFANCIA TEMPRANA. ESTUDIO DE CASOS
}

\author{
Fostering preschool children's social-emotional competence. Case studies
}

Natalia Ibáñez-Martínez y Clara Romero-Pérez

Fecha de recepción: 01/12/2019

Fecha de aceptación: 15/12/2019

RESUMEN: El desarrollo de la competencia socioemocional constituye un objetivo central en la etapa escolar. Dada su importancia en todas las etapas educativas y, especialmente en Educación Infantil, el profesorado diseña programas con el fin de desarrollar estas habilidades en el alumnado. El estudio que se presenta persigue como objetivo conocer cómo el profesorado planifica e implementa programas que promueven la competencia socioemocional en la etapa infantil. La metodología empleada, de corte cualitativo, se ha apoyado en el estudio de casos, tomando como casos al profesorado participante de dos centros educativos públicos de Andalucía en los que se estaban llevando a cabo sendos programas dirigidos al desarrollo de la competencia socioemocional durante el curso 2018/2019. Las técnicas empleadas fueron la entrevista no estructurada, realizada en cada centro a la persona coordinadora del programa y a una tutora de Educación Infantil que lo estuviese implantando y el análisis de los programas implementados. Los resultados de la investigación muestran coincidencias en ambas propuestas de intervención: objetivos, contenidos, requisitos para su implantación e impacto percibido. Las diferencias se dieron en las limitaciones percibidas por el profesorado sobre las acciones ejecutadas.

PALABRAS CLAVE: competencia socioemocional; educación emocional; educación infantil; estudio de casos.

ABSTRACT: Social-emotional competence has been a central goal in school education. Given its importance in all educational stages and, especially in Early Childhood Education, teachers design programs for students to enhance or develop it. This research has been developed to offer an overview of the way teachers plan and implement programs fostering the emotional competence in early childhood. A literature review and a subsequent qualitative study of multiple cases were performed. Two public schools in Andalusia with an educational project aimed at developing social-emotional competence was being carried out during the academic year 2018/2019 participated. The techniques used were the unstructured interviews to the coordinator of the program and a Pre-school Education tutor $w$ and the analysis of the programmes' documentation. Results show coincidences between both intervention proposals regarding objectives, contents, requirements for their implementation and perceived impact.; however, there were differences in the perception of limitations perceived by the teaching staff on the actions implemented.

KEY WORDS: social-emotional competence; emotional education; early childhood education; case studies. 


\section{Introducción}

El período preescolar representa una oportunidad única para promover el desarrollo social y emocional en la primera infancia. Entre los 2 y 7 años se desarrolla la capacidad para regular y expresar las emociones, y se exhiben habilidades sociales para afrontar las emociones propias y ajenas. Uno de los dominios de aprendizaje relevantes en estas edades es el socioemocional. Promover entornos de aprendizaje de calidad que contribuyan a desarrollar en estas edades una adecuada percepción de sí mismos y de los demás, entablar relaciones positivas con sus iguales y los adultos, ser sensibles a las necesidades de los otros, y aumentar el uso de habilidades socio-emocionales para afrontar las emociones propias y ajenas son dimensiones educables que no han de desdeñarse en la educación de la primera infancia. La educación emocional proporciona una buena parte de estos aprendizajes (Elias, 2003).

Las investigaciones sobre la efectividad de las intervenciones educativas en el ámbito socio-emocional han demostrado que el impacto de estas actuaciones es positivo para la salud mental y la construcción de la personalidad. Numerosos estudios han demostrado el impacto de la educación emocional en la primera infancia (McCabe y Altamura, 2011; Bierman y Motamedi, 2015; Schindler et al., 2015; Sabey et al., 2017; Gershon y Pellitteri, 2018; Kemple, Lee y Ellis, 2019). El metaanálisis realizado por Blewitt, Fuller-Tyszkiewiez, Nolan et al. (2018) sobre la efectividad de los programas dirigidos al desarrollo de la dimensión socioemocional en la edad preescolar concluye que el mayor efecto de estos programas se produce en la mejora de la competencia socioemocional, o lo que es lo mismo, en la capacidad para regular, expresar y controlar las emociones. Asimismo, concluye que junto con estos programas, el establecimiento de relaciones de apego seguras con los adultos y el apoyo social que se brinde contribuyen, asimismo, a mejorar la competencia socioemocional en la primera infancia (Blewitt, Morris, Nolan et al., 2018).

\section{Educar la competencia socioemocional}

Como se ha señalado, la competencia socioemocional se construye a lo largo del desarrollo evolutivo y conforma una capacidad útil para afrontar las situaciones vitales. Esta competencia, de acuerdo con los modelos propuestos por Bisquerra y Pérez (2007), PérezEscoda, Filella, Alegre y Bisquerra (2012), CASEL (2013) se compone de cinco dimensiones: conciencia emocional, regulación emocional, autonomía personal/autorregulación (CASEL), competencia social y competencias para la vida y el bienestar/toma de decisiones responsables (CASEL). Aunque fundamental en todas las etapas educativas, en educación infantil es especialmente importante, debido a que es en los primeros años de vida cuando se comienzan a desarrollar habilidades que les permiten captar la información que las emociones transmiten y operar con ellas. Asimismo, en esta etapa se producen en los niños y las niñas numerosas vivencias emocionales relacionadas con la familia, los iguales, la escuela, etc., que brindan la oportunidad de establecer las bases de su competencia emocional futura.

A través de acciones educativas dirigidas a desarrollar la dimensión emocional, se dota a los escolares de habilidades y estrategias emocionales que cumplen una valiosa función protectora de factores de riesgo (De Andrés, 2005; Bisquerra, 2003). Los beneficios de este tipo de educación (Durlak, Weissberg, Dymnicki, Taylor y Schellinger, 2011; Bothe, Grignon y Olness, 2014) se traducen en mejoras significativas en la inteligencia emocional, comportamientos sociales positivos, mayores actitudes adaptativas, disminución de problemas conductuales y reducción de la angustia emocional, entre otros. Por este motivo, han surgido diversos programas elaborados por el profesorado con el fin de desarrollar la competencia socioemocional en la primera infancia (Kress y Elias, 2007; Pahl y Barret, 2007; Stefan, 
2018). La información sobre las dinámicas de implementación de programas de educación emocional por parte del profesorado es escasa (O’Connor, Dyson, Cowdell y Wtason, 2017) y, muy especialmente, en los programas dirigidos a la primera infancia. La investigación que se presenta pretende aportar datos que cubran estas lagunas de conocimiento.

\section{Recomendaciones para la elaboración de programas para movilizar la competencia socioemocional}

A pesar de que no existe un consenso acerca de los aspectos que pueden determinar el éxito en la elaboración de programas que incidan en la competencia socioemocional, la literatura científica aporta algunas claves. Desde el punto de vista del diseño, Goetz, Frenezel, Pekrun y Hall (2005) indican la existencia de dos enfoques dentro de la educación emocional: el restringido, vinculado al desarrollo exclusivo de la inteligencia emocional y el laxo, en el que se propicia la ejecución de programas que fomenten las habilidades de toma de decisiones, de modificación cognitivo-conductual o de habilidades sociales. Sin tener en cuenta el patrón de intervención, Zeidner, Roberts y Matthews (2008) subrayan la importancia de establecer un marco teórico concreto que asiente las bases de las experiencias educativas.

Respecto a los objetivos generales de estos programas, aunque dependerán del enfoque o teorías en las que se apoyen las intervenciones, Bisquerra (2010) enumera los siguientes: a) identificar y reconocer las emociones propias y las de los demás, b) controlar las propias emociones, c) prevenir los efectos de las emociones negativas, d) desarrollar la habilidad de generar emociones positivas, e) adoptar una actitud positiva ante la vida, entre otros.

En base a estos, el mismo autor (Bisquerra, 2003) establece que los contenidos que deben estar presentes en estos programas son la conciencia sobre emociones propias y de los demás, la motivación, la regulación de las emociones, las habilidades socio-emocionales o el bienestar social.

En relación con la disposición temporal de las acciones, se ha demostrado que los proyectos que logran mejores resultados son los que comienzan por la percepción, siguen por la comprensión y finalizan con la regulación emocional (Durlak et al., 2011). En cuanto a la evaluación de los programas, Álvarez, Bisquerra, Fita, Martínez y Pérez (2000) y Pérez (2006) estiman que debe constatarse la adquisición de conocimientos básicos sobre emociones y las competencias emocionales, así como los estados emocionales. Además, recomiendan que este proceso lo lleve a cabo el profesorado junto a una persona asesora profesional de psicología y pedagogía; empleando para ello más de un instrumento que combine datos cuantitativos y cualitativos. Para que se realice de forma adecuada, la temporalización de la evaluación debe darse en tres momentos: antes de la implantación del programa analizando las necesidades del alumnado y el programa en sí mismo, durante y después del mismo evaluando el proceso y el producto.

En relación con la efectividad de los programas, se considera que estos lo lograrán cuando utilicen un enfoque basado en la capacitación con pasos secuenciales, el aprendizaje sea activo, el tiempo que se le dedique sea el suficiente para el desarrollo de habilidades y tengan objetivos de aprendizaje explícitos (Durlak et al., 2011). Asimismo, Graczyk et al. (2000) indican como clave indispensable para el éxito la formación previa a la implementación del curso, ya sea a través de guías y/o personas expertas en este ámbito. En esta misma línea, Bisquerra (2010) y Blewitt, Morris, Nolan et al. (2018) subrayan la gran relevancia que tiene la implicación de la comunidad educativa en la planificación y el desarrollo del programa. No 
es suficiente con que el cuerpo docente se implique y se forme, sino que es necesaria la colaboración de las familias, al mismo tiempo que es necesario adoptar una mirada holística y sostenible en las fases del diseño, planificación e implementación de las propuestas de actuación (Martinsone, 2016).

\section{Método}

La investigación presentada se ha apoyado en un enfoque cualitativo, con el fin de estudiar la realidad que experimentan las personas participantes para comprenderla, teniendo en cuenta su contexto personal y sus perspectivas (Quecedo y Castaño, 2002; Romero y Ordóñez, 2018). Asimismo, se ha empleado una metodología inductiva, en la que se ha partido de unos casos concretos, con el objetivo de descubrir relaciones y analizar de manera profunda la situación (Stake, 2010; Romero y Ordóñez, 2018).

El diseño empleado es un estudio de casos que ha permitido explorar aquellos aspectos que no se encuentran especificados en las memorias de los programas de educación emocional difundidas en el repositorio Averroes (Junta de Andalucía) https://bit.ly/30Qjx9r. Los casos, profesorado no universitario involucrado en el diseño e implementación de proyectos para la mejora de la competencia emocional en la etapa infantil, se han seleccionado teniendo en cuenta tres criterios: i) que impartieran docencia en el segundo ciclo de Educación Infantil; ii) que participaran de modo activo en proyectos que hubieran sido aprobados por la Consejería de Educación, Junta de Andalucía y, iii) que los proyectos se estuvieran desarrollando durante el curso escolar 2018-2019.

\subsection{Objetivos}

El principal objetivo del presente estudio de casos es analizar cómo el profesorado de la etapa de Educación Infantil ha planificado y desarrollado programas para el fomento de la competencia socioemocional dirigidos a escolares en edad preescolar. Se han formulado los siguientes objetivos específicos:

- Comparar los programas de desarrollo de la competencia socioemocional implementados en los dos centros educativos analizados.

- Identificar las motivaciones y los requisitos necesarios para la implantación y el desarrollo de un programa de Educación Emocional dirigido a la Etapa Infantil, a partir de la voz del profesorado participante.

- Conocer el impacto percibido que ha tenido el programa sobre el alumnado y el profesorado a partir de las valoraciones de las personas informantes.

- Sistematizar las dificultades encontradas y las propuestas de mejora sobre los programas implementados.

\subsection{Población y Muestra}

En la presente investigación, el caso queda delimitado por el profesorado, individualmente considerado, que ha participado activamente en el diseño y desarrollo de los programas para la mejora de la competencia socioemocional de los escolares en el segundo ciclo de educación infantil, siendo una la maestra que está ejecutando el proyecto en su aula de Educación Infantil y la otra persona, la coordinadora de este. De acuerdo con los criterios de elegibilidad que se han señalado anteriormente, han participado en el estudio cuatro docentes involucrados en el curso 2018/2019 en sendos programas: 9 meses, 9 emociones 
(9M9E), titulado inicialmente como Serendipia: buscando dentro de ti, y Educar desde la emoción (EDE).

En total, los casos comprenden tres profesoras y un profesor, cuyas edades se encuentran comprendidas entre los 33 y los 48 años. Asimismo, la totalidad de las personas participantes cuentan con una experiencia docente superior a 10 años y llevan trabajando en sendos centros durante, al menos, dos años continuados. Los centros en los que se han desarrollado ambos proyectos corresponden al CEIP Rafael Alberti (Dos HermanasMontequinto, Sevilla) y Colegio Público Rural Agrupado (CPR) Monte Chullo (Huéneja, Granada). Ambos centros poseen una dilatada experiencia liderando proyectos de innovación educativa adscritos a los diferentes Planes y Programas de la Consejería de Educación de la Junta de Andalucía. Entre ellos, Red Andaluza Escuela "Espacio de Paz" y Promoción de Hábitos de Vida Saludable, a cuyas líneas estratégicas se vinculan los programas implementados por las personas informantes.

El tipo de muestra seleccionada es dirigida, homogénea, basada en casos tipo. Para conformar los casos se ha tenido en cuenta la accesibilidad, la adecuación de los participantes al perfil propio del objeto de estudio y que mostraran una predisposición positiva hacia el mismo. De un total de 6 proyectos incentivados, sólo 4 satisfacían los criterios de elegibilidad para el estudio y únicamente el profesorado de 2 centros accedió a participar en el estudio. Aun cuando se trata de una muestra con una baja representatividad estructural, tiene la función de ser una investigación exploratoria de la situación actual y de inicio de un estudio ulterior (Yin, 2009; Hernández, 2015). De acuerdo con el tipo de estudio llevado a cabo, estudio de casos en profundidad, el tamaño mínimo de la muestra se ajusta a las recomendaciones señaladas por Creswell (2014) y Hernández Sampieri, Fernández Collado y Baptista Lucio (2014) para este tipo de estudios cualitativos: de tres a cinco casos.

\subsection{Instrumento}

La recogida de información se ha realizado a partir de la técnica de la entrevista y el análisis de los programas implementados. A través de la entrevista se ha podido conocer la perspectiva de las personas participantes, el significado de sus conductas, las actividades que realizan, los objetivos que pretenden alcanzar, etc. (León y Montero, 2015). Se ha realizado en profundidad y es no estructurada, por tanto, las preguntas planteadas tienen un carácter abierto para que la persona entrevistada pueda expresar sus opiniones y experiencias de la manera que considere oportuna (Carbonero y Caparrós, 2015).

Los temas y preguntas que se han efectuado en la entrevista son de elaboración propia y están basados en una previa búsqueda bibliográfica ejecutada con el fin de identificar los aspectos más relevantes para la investigación. Todo ello siguiendo el modelo propuesto por León y Montero (2015).

\subsection{Procedimiento de recogida y análisis de datos}

Previamente a la realización de las entrevistas, se contactó con los centros por vía telefónica, se les explicó la finalidad de la presente investigación y los temas sobre los que se deseaba indagar, tal y como recomiendan Rodríguez, Gil y García (1996). Estas se realizaron de manera presencial o telefónica, pero siempre desarrollándose en un lugar que preservase su intimidad. En ambos casos, se les pidió que diesen su consentimiento para ser grabadas. 
Una vez se obtuvieron todas las entrevistas, se estableció un código para cada una de ellas, se transcribieron y se efectuó una comprobación con la muestra sobre la información que habían proporcionado en la que ninguna quiso realizar cambios. Después, con el software Weft QDA, se llevó a cabo la reducción, disposición y transformación de los datos a partir de unos sistemas de categorías descriptivas de carácter deductivo.

\section{Resultados}

\subsection{Estructura del programa}

En este apartado se han analizado seis elementos de los programas de educación emocional implementados: i) modelos teóricos; ii) objetivos; iii) contenidos; iv) temporalización y sostenibilidad; v) metodología y vi) evaluación.

Las principales diferencias entre los programas llevados a cabo se refieren a los cuatro primeros elementos anteriormente mencionados (Tablas 1-4).

Tabla 1. Modelos teóricos de los programas de educación emocional implementados

9M9E EDE

Basado en la Teoría de las Inteligencias Múltiples

"Lo que hemos hecho ha sido mirar, buscar información y, a raíz de ahí, lo hemos adaptado a nuestro centro, al profesorado, a las iniciativas que hemos tenido unos y otros y a las necesidades de los niños $[\ldots]$ pero es una mezcla de todo" (9M9E, 40)
"Estas capacidades [inteligencia intra e interpersonal] ya se encuentran en nuestros alumnos y, a partir del trabajo en el colegio, pueden ir desarrollando hasta lograr resolver problemas por ellos mismos y otros aspectos que ya te he comentado" (EDE, 33)

Tabla 2. Objetivos de los programas de educación emocional implementados

\section{M9E}

Identificar sus emociones, regular su respuesta emocional y generar respuestas positivas a sus emociones.

"Tomar conciencia de las emociones que nos ayudan a vivir en la vida y en la sociedad, sabiéndolas identificar, comprender y expresar" (9M9E, 48).
Conocer sus emociones y las de los demás, regular su respuesta emocional, aprender a convivir con sus iguales, empatizar con el resto y generar respuestas positivas a sus emociones.

"Saber identificar cómo se sienten así, qué es lo que provoca que se sientan así, cómo poder resolver la situación o cómo actuar para que eso no pase" (EDE, 39). 
Tabla 3. Contenidos de los de los programas de educación emocional implementados

9M9E EDE

Conocimiento y regulación emocional, Conocimiento de las emociones propias y las de habilidades sociales y resolución de conflictos los demás, regulación emocional, empatía, ayuda entre iguales, autonomía, conocimiento de sí mismo, y convivencia entre iguales.

"Tienen que conocerse a ellos mismos y tomar conciencia de sus emociones, pero también tienen que saber expresarlas [...], conocer las emociones de los demás y entenderlas" [9M9E, 48].
"Que sean felices, pero no feliz de decir qué feliz estoy, sino de que valoren, sean conscientes de sus habilidades y de sus capacidades, escuchen lo que sucede al otro y piensen en cómo ayudarle" (EDE, 39).

Tabla 4. Duración y sostenibilidad de los de los programas de educación emocional implementados

\section{M9E}

1 curso escolar (2018/2019). 6-7 horas al mes.

\section{Desean continuar}

"Teniendo en cuenta los buenos resultados, seguramente, continuaremos con él" [9M9E, 40].

"6-7 horas al mes, además del tiempo que quiera dedicarle cada tutor/a. Ya no solo las actividades que se generan a nivel de centro, sino como también luego cada tutor en su aula adapta la emoción que estamos trabajando y lo desarrolla también en su aula [...] el tiempo depende también de la temática que se haya trabajado" [9M9E, 40].

\section{EDE}

2 cursos escolares (2017-2019). 1 sesión semanal.

\section{Desean continuar}

"Esto no es algo que haya empezado y tenga su fin. Aún no hemos realizado los papeles para la Junta, pero por falta de tiempo por temas burocráticos... pero nosotros pensamos continuar trabajando de esta forma" [EDE, 33].

"Realizan una sesión por semana, además del tiempo que quiera dedicarle cada tutor/a. [...] realmente se trabaja más porque cuando los niños vuelven del patio y ha sucedido algún problema o alguno está enfadado, nos sentamos a hablar de ello, de las emociones que sienten, de las relaciones entre ellos..." [EDE, 39].

Las similitudes de ambos programas se reflejan en la metodología y en la evaluación. Respecto de la primera, tanto en el proyecto $9 M 9 E$ como en $E D E$, se emplean diversas estrategias para acercar los contenidos al alumnado. Algunas de ellas son los cuentos, la relajación, las técnicas plásticas, las dinámicas, rol-playing o los juegos manipulativos. Al mismo tiempo, en ambos programas se destacó la importancia de hacer partícipe al alumnado 
de su propio aprendizaje: "metodología activa, motivadora, en la que el niño sobre todo tome conciencia, participe" (9M9E, 48).

Con relación a la evaluación, se realizó en diferentes momentos, comenzando por el análisis de las necesidades del alumnado y continuando con el estudio de su implantación y de sus resultados. Este último tipo de evaluación, aún pendiente de su ejecución en los dos grupos, será realizada por la persona coordinadora y se efectuará mediante la observación directa del alumnado. Además, en la experiencia 9M9E, también se tendrá en cuenta la satisfacción de las familias y la del personal docente.

\subsection{Génesis de los programas y requisitos necesarios para su implantación}

Atendiendo al origen de los programas de educación emocional, los dos equipos docentes coincidieron expresando que antes de su implantación apenas se educaba en este ámbito al alumnado, ya que dependía de cada docente. Asimismo, ambos mostraron que consideraban la educación emocional un plano a desarrollar en el alumnado. Además, en el proyecto 9 meses, 9 emociones, las profesoras añadieron que es fundamental comenzar a trabajarlas desde la etapa de Educación Infantil porque: "es cuando los niños están construyendo su mundo emocional, es cuando realmente necesitan identificar las emociones, conocerlas, aprender a manejarlas..." (9M9E, 40). Continuando con el origen del programa, el profesorado de $E D E$ indicó que, antes de planificarlo, llevaron a cabo un proyecto de emociones en la etapa de Educación Infantil y ante los exitosos resultados que obtuvieron, consensuaron seguir trabajando las emociones y extenderlo también a Educación Primaria.

En cuanto a los requisitos necesarios para la implantación de los programas, destacan la formación, los recursos materiales y los agentes implicados o recursos personales. En ambas experiencias se ha expresado que la formación fue uno de los elementos imprescindibles tanto para su planificación como para su implantación. En las dos, el profesorado realizó varios cursos sobre inteligencia emocional o educación emocional, los más destacados fueron impartidos por Rafael Bisquerra en el caso de $9 M 9 E$ y por Alberto Ortega en $E D E$.

Respecto a los recursos materiales adquiridos, fueron posibles gracias a la dotación de financiación por parte de la Junta de Andalucía. Ambos grupos coincidieron en que esta subvención fue necesaria para la compra de materiales, más específicamente, para la adquisición de una amplia biblioteca de educación emocional que sirviese tanto para el cuerpo docente como para el alumnado de todos los niveles y materiales fungibles requeridos para el desarrollo de las actividades.

En relación con los agentes implicados, ambas parejas de participantes coincidieron en que para ellos/as fue muy importante la participación y colaboración de las familias. Sin embargo, cada programa tiene un enfoque diferente. Mientras que en $9 M 9 E$ buscaban que las familias participaran en las actividades que realizaron dentro de las aulas, en el centro del programa $E D E$ establecieron la participación de estas a partir de una escuela de familias externa al horario lectivo.

\subsection{Impacto percibido de los programas implementados}

Los resultados de la implantación de los dos programas de educación emocional se extienden entre el alumnado, el profesorado y la dinámica de aula. El equipo docente de los 
dos programas afirmó haber observado cambios positivos en todos ellos, aún sin haberlos finalizado.

En cuanto al impacto percibido sobre el alumnado, ambos grupos expresaron que, en la actualidad, las relaciones entre los/as propios/as estudiantes han mejorado, por ejemplo, "se cuidan más entre los propios niños y sin importar si son amigos o son de otra clase" (EDE,39). Asimismo, las participantes del programa 9 meses, 9 emociones indicaron beneficios en sus competencias, como saber reconocer emociones y gestionarlas. Por su parte, en el otro programa se han mencionado competencias como expresar emociones de manera gestual y oralmente; así como la disminución de estudiantado conflictivo a partir de la implantación de este. En esta misma línea, el personal docente resaltó el hecho de que las familias les informaron sobre estas mejoras.

Con relación a los beneficios que afectan al profesorado, el programa $E D E$ expuso que estos han sucedido a dos niveles: personal y profesional. En el personal, destacaron beneficios como conectar consigo mismo/a y mejorar la empatía o el pensar en todas las personas; mientras que, a nivel profesional, se centraron en la unión que ha supuesto estructurar un programa de estas características. Por otro lado, ambos grupos mencionaron que ha aumentado la implicación del profesorado al ver los beneficios que estaban obteniendo. En este sentido, el profesor del proyecto Educar desde la emoción indicó que el personal docente se encontraba más cómodo en el centro por el trabajo que estaban desempeñando y, por ello, deseaban seguir trabajando en él:

Antes en el colegio había mucho profesor itinerante [...] Era un centro donde no se quería quedar nadie y desde el cambio directivo ya hay muchos profesores que llevamos aquí muchos años y tenemos pensado continuar, por lo que si se están quedando será que hay cosas que se están haciendo bien y por ello quieren seguir trabajando aquí. (EDE, 33)

Finalmente, respecto al impacto sobre la dinámica de aula, mientras que en el proyecto 9M9E las profesoras expresaron que ha mejorado la relación entre los/as docentes y el alumnado, así como el clima del aula destacando aspectos como el afecto y la autoestima de los niños y las niñas; en la experiencia $E D E$ destacaron la mejora del comportamiento del alumnado, que provoca que haya menos conflictos, y la reducción de la reactividad de los chicos y las chicas, mostrando sus preferencias y sentimientos antes que actuar de manera agresiva (pegando).

\subsection{Expectativas, limitaciones y mejoras}

Las participantes de $9 M 9 E$, no esperaban que este tuviese tanta repercusión y se obtuviesen unos beneficios tan notables a corto plazo. Mientras que, en el otro proyecto, consideraban que sí iba a tener ese éxito y, por ello, estaban tan emocionados y emocionadas desde el primer momento.

En cuanto a las limitaciones que han encontrado al planificar y ejecutar los programas, en el $9 M 9 E$ destacaron una menor participación de las familias de lo que deseaban y la enorme dificultad que supuso realizar una experiencia que influyó a tres centros por ser un colegio rural agrupado:

Se ha tenido que hacer en la misma localidad tres o cuatro veces, pasar a otra localidad y otra localidad... cuando a lo mejor la persona que lo hace... por ejemplo si yo lo tengo que 
hacer, tengo que ir a las tres localidades, tienen que estar pendientes de cuando yo vaya... (9M9E, 40)

En el proyecto $E D E$, mencionaron como limitaciones la falta de organización del primer año, ya que parte del profesorado no empleaba la hora de tutoría para trabajar la inteligencia emocional. Asimismo, también indicaron que la formación necesaria dificultaba el proceso porque no todo el personal docente podía asistir a los cursos por su amplia duración o el horario que tenían.

Respecto a las mejoras que proponían de cara a la continuación de los programas, todos los participantes entrevistados coincidieron en que buscarían la forma de implicar más a las familias y que no cambiarían los programas debido a que ya los habían modificado atendiendo a sus necesidades iniciales y a las que fueron surgiendo. Por tanto, consideran que el proyecto debería seguir creciendo como lo había hecho hasta ahora: "Realmente nosotros ya hemos ido cambiando aquellas cosas que veíamos que no funcionaban [...] Siempre habrá cosas que mejorar y en eso trabajamos día a día” (EDE, 39).

\section{Discusión y conclusiones}

La presente investigación tiene como objetivo principal analizar cómo el profesorado de Educación Infantil ha planificado e implementado programas de educación emocional. Con el fin de alcanzar el mencionado objetivo, se plantearon una serie de objetivos específicos para estudiar más en profundidad el estado de la cuestión.

En cuanto al primer objetivo específico, comparar la estructura de los programas de educación emocional, se puede afirmar que tanto el programa $9 M 9 E$ como $E D E$ comparten de manera estructural los mismos elementos, coincidiendo en el contenido de muchos de ellos.

Más concretamente, ambas experiencias parten del entendimiento de la inteligencia emocional como habilidad (Salovey y Mayer, 1990) o como corriente mixta (Bar-On, 2006); debido a que los dos proyectos buscan fomentar o potenciar la competencia socioemocional en su alumnado. Sin embargo, en los dos programas el equipo docente se mostró dudoso ante la pregunta sobre qué teorías se encontraban basados y mientras que, en $E D E$ finalmente explicaron que seguían la Teoría de las Inteligencias Múltiples, en el programa $9 M 9 E$ reconocieron no ceñirse a ninguna teoría o modelo concreto, acción desaconsejada por Zeidner et al. (2008).

Respecto a los objetivos, ambas experiencias dieron prioridad a la identificación de las emociones, la regulación de la respuesta emocional y la generación de respuestas positivas en relación con sus emociones. Todos ellos se encuentran dentro de los explicitados por Bisquerra (2010); pero, además, el programa $E D E$ añade otros elementos que coinciden con este autor, como la identificación de las emociones de los demás. Asimismo, ambos proyectos coinciden con Bisquerra (2003) en los contenidos planteados, aunque aportando algunos matices diferentes como la resolución de conflictos $(9 M 9 E)$, la autonomía o la convivencia entre iguales $(E D E)$. Cabe destacar que, en ambas experiencias, tal y como aconseja este autor (2003), los mencionados contenidos fueron adaptados a la edad del alumnado y a sus conocimientos previos, atendiendo a su nivel educativo. Siguiendo con las indicaciones propuestas por Bisquerra (2003), este concreta una metodología práctica. Esta se ha visto reflejada en los dos programas, ya que estos planteaban la necesidad de realizar actividades de diferente índole (cuentos, relajaciones, role-playing...) haciendo partícipe a todo el alumnado. 
De esta manera, la metodología que han aplicado puede describirse como motivadora, participativa y activa (Durlak et al., 2011).

Otra de las similitudes relativa a la estructura entre los dos casos era la disposición de los contenidos. Tanto en $9 M 9 E$ como en $E D E$, el personal docente mencionó los contenidos en el mismo orden que proponen Durlak et al. (2011) para la obtención de mejores resultados, es decir, comenzaron por la percepción, continuaron con la comprensión de las emociones y finalizaron con la regulación de estas. Asimismo, para la adquisición de los conocimientos, estos mismos autores indicaron que para asegurar la efectividad de los programas, el tiempo dedicado a ellos debía ser suficiente. No obstante, en ninguno de los dos proyectos se concretan las horas totales que se implementa porque, pese a haber un tiempo establecido, ambos programas mencionan que existen otros momentos en los que el propio profesorado debe trabajarlos. Por ende, el tiempo que se le dedica dentro del aula dependerá de cada docente: importancia que le otorgue, motivación ante la tarea, tiempo del que disponga, facilidad de relación de la educación emocional con los contenidos propios de su materia, etc.

El último aspecto en común es la evaluación de la adquisición de competencias y resultados obtenidos con la aplicación de los programas. Tanto en $9 M 9 E$ como en $E D E$, se ha llevado a cabo una estimación de los conocimientos sobre las emociones, las competencias emocionales que presentaba el alumnado y sus estados emocionales; junto a una evaluación del programa, de su implantación y de los resultados que estaban obteniendo durante y tras su desarrollo, tal y como recomiendan Álvarez et al. (2000) y Pérez (2006). Sin embargo, en ambos casos los programas solamente se evaluaban por el encargado o la encargada de coordinación mediante la observación directa. Es decir, estaban utilizando un instrumento de índole cualitativa y a una única persona, aspectos que no coinciden con las instrucciones de Álvarez et al. (2000) y Pérez (2006) que, señalan que deben aplicarse instrumentos cualitativos y cuantitativos, utilizados por diferentes profesionales pertenecientes al ámbito de la psicología y de la pedagogía, además del profesorado, con el fin de extraer la máxima información posible para asegurar la fiabilidad y validez de la evaluación.

El segundo objetivo específico busca identificar las motivaciones y los requisitos necesarios para la implantación y el desarrollo de un programa de educación emocional. Tanto en el proyecto $9 M 9 E$, como en $E D E$, se consideró importante el desarrollo de la educación emocional en la etapa del segundo ciclo de Educación Infantil y se estimó que no se estaba trabajando de forma correcta, ya que como se ha mencionado anteriormente, el tiempo que se le dedicaba dependía de cada docente. Además, ambos expresaron que el requisito más necesario para la implantación de los programas fue la formación, junto a los recursos materiales y personales. En el caso de la formación, el profesorado de ambos centros realizó varios cursos con expertos en esta área y adquirieron manuales de formación para la biblioteca escolar. Las acciones descritas coinciden con las indicadas por Graczyk et al. (2000), que las califica como fundamentales para garantizar el éxito de los programas. Por otro lado, en cuanto a los recursos personales, los dos centros concuerdan con Bisquerra (2010) en que la familia es un factor indispensable para los programas. No obstante, se ha podido observar que mientras que en $9 M 9 E$ han intentado que estas formen parte de su proyecto colaborando en el desarrollo de diferentes actividades, en $E D E$ las han incluido, pero de forma externa; es decir, han creado un espacio solamente para ellas que se caracteriza por la inexistencia de influencia directa sobre el propio programa.

En función del tercer objetivo específico, conocer el impacto percibido que ha tenido el programa sobre el alumnado y el profesorado a partir de las valoraciones de este último colectivo, el cuerpo docente de ambas escuelas afirmó que estaban observando importantes 
beneficios. Algunos de estos tienen repercusión en el alumnado y coinciden con los expuestos por Durlak et al. (2011) como, por ejemplo, la mejora en los logros de competencia socioemocional, la disminución de problemas conductuales y la aparición de comportamientos sociales más positivos. Sin embargo, también es importante destacar que ambos equipos docentes expresaron haber obtenido beneficios referentes a la mejora del clima del aula o a la implicación del cuerpo docente en su desempeño profesional.

Finalmente, en cuanto al cuarto y último objetivo específico, recoger las dificultades y las propuestas de mejora de los programas, las profesoras de $9 M 9 E$ expresaron que las dificultades encontradas versaban sobre el organigrama del propio centro por estar dividido en tres localidades y sobre la escasa implicación de la familia. Por tanto, como ya se había mencionado anteriormente, coinciden con Bisquerra (2010) en el papel vital que le otorgan a esta. No obstante, en el programa $E D E$ resaltan como limitación la falta de implicación de parte del profesorado debido a que en un primer momento no cumplían con el horario establecido para el desarrollo de la educación emocional. En lo referente a las mejoras, el profesorado que lideraba ambos proyectos coincidieron en que iban a buscar la forma de implicar más a la familia y en que realizaban un proceso de mejora continuo en el que efectuaban los cambios que consideraban oportunos durante su desarrollo. Por tanto, se puede deducir que ambos proyectos, tal y como se ha comentado, han llevado a cabo evaluaciones de estos y de sus resultados durante su desarrollo, y han efectuado cambios con el fin de lograr los objetivos de aprendizaje que habían propuesto (Durlak et al., 2011).

Por ende, pese a que los programas pareciesen muy diferentes a primera vista, se ha podido constatar cómo los elementos de los que disponen son similares. Asimismo, ambas experiencias han llevado a cabo numerosas prácticas aconsejadas por autores y autoras y por ello, podrían ser calificadas como buenas prácticas.

En relación con las limitaciones encontradas durante la elaboración de la investigación, destaca el reducido número de centros de Educación Infantil en toda Andalucía que contaran, durante el curso 2018/2019, con un proyecto de innovación educativa aprobado por la Consejería de Educación (Junta de Andalucía) y que estuviese centrado en el desarrollo de la competencia socioemocional en Educación Infantil. Como se ha señalado, de los cuatro programas aprobados, la mitad de los centros educativos que conformaban la muestra inicial, declinaron participar en la presente investigación.

Por ende, se plantea la siguiente propuesta para futuras investigaciones: realizar un estudio de casos múltiple más amplio, incluyendo un número mayor de programas de educación emocional de carácter internivelar. Además, sería enriquecedor emplear como instrumento complementario la observación directa para enriquecer y contrastar las percepciones del profesorado con la realidad de las aulas. Los resultados de la investigación propuesta tendrían el fin de determinar acciones futuras como, por ejemplo, que las administraciones educativas elaboren una Guía de Buenas Prácticas para el desarrollo de programas de educación emocional o formación específica para los/as profesionales docentes, en los que se incida en la estructura de estos programas y se especifiquen pautas que garanticen su éxito.

\section{Referencias bibliográficas}

Álvarez, M., Bisquerra, R., Fita, E., Martínez, F. y Pérez, N. (2000). Evaluación de programas de educación emocional. Revista de Investigación Educativa, 18 (2), 587-599. Recuperado de https://bit.ly/36xVwHB 
Bierman, K. L., y Motamedi, M. (2015). SEL programs for preschool children. In J. A. Durlak, C. E. Domitrovich, R. P. Weissberg, y T. P. Gullotta (Eds.), Handbook on social and emotional learning: Research and practice (pp. 135-150). New York, NY: The Guilford Press.

Bisquerra, R. (2003). Educación emocional y competencias básicas para la vida. Revista de Investigación Educativa, 21(1), 7-43. Recuperado de https://bit.ly/38FF89K

Bisquerra, R. (2010). Educación emocional y bienestar. Madrid: Wolsters Kluwer España S.A.

Bisquerra, R. (2012). Diversidad y escuela inclusiva desde la educación emocional. En J. Navarro, Diversidad, calidad y equidad educativas (pp. 24-32). Murcia: Consejería de Educación, Formación y Empleo.

Blewitt, C., Fuller-Tyszkiewicz, M., Nolan, A., Bergmeier, H., Vicary, D., Huang, T., ... Skouteris, H. (2018). Social and Emotional Learning Associated With Universal Curriculum-Based Interventions in Early Childhood Education and Care Centers. JAMA Network Open, 1(8), e185727. doi: 10.1001/jamanetworkopen.2018.5727

Blewitt, C., Morris, H., Nolan, A., Jackson, K., Barrett, H., y Skouteris, H. (2018). Strengthening the quality of educator-child interactions in early childhood education and care settings: a conceptual model to improve mental health outcomes for preschoolers. Early Child Development and Care, 1-14. doi.org/10.1080/03004430.2018.1507028

Bothe, D. A., Grignon, J. B., y Olness, K. N. (2014). The Effects of a Stress Management Intervention in Elementary School Children. Journal of Developmental y Behavioral Pediatrics, 35(1), 62-67. doi:10.1097/dbp.0000000000000016

Carbonero, D. y Caparrós, N. (2015). La entrevista como técnica de investigación. En N. Caparrós y E. Raya. Métodos y técnicas de investigación en trabajo social (pp.155174). Madrid: Grupo 5.

Collaborative for Academic, Social, and Emotional Learning (CASEL) (2013). Effective social and emotional learning programs: Preschool and elementary school edition. Chicago, IL: CASEL. Recuperado de https://bit.ly/36z7pgs

Creswell, J.W. (2014). Research design: qualitative, quantitative, and mixed methods approaches, $4^{\mathrm{a}}$ ed. Thousand Oaks, CA:Sage Publications.

Durlak, J.A., Weissberg, R.P., Dymnicki A.B, Taylor R. D. y Schellinger, K. B. (2011). The Impact of Enhancing Students' Social and Emotional Learning: A Meta-Analysis of School-Based Universal Interventions. Child Development, 82 (1), 405-432. doi: $\underline{10.1111 / \mathrm{j} .1467-8624.2010 .01564 . \mathrm{x}}$

Elias, M. (2003). Aprendizaje Académico y Socio-Emocional. Bruselas/Ginebra: Academia Internacional de Educación y Oficina Internacional de Educación. Recuperado de https://bit.ly/38HTVky 
Gershon, P. y Pellitteri, J. (2018). Promoting Emotional Intelligence in Preschool Education: a review of programs. International Journal of Emotional Education, 10(2), 26-41. Recuperado de https://bit.ly/2PjwExx

Goetz, T., Frenzel, A.C., Pekrun, R. y Hall, N. (2005). Emotional Intelligence in the context of learning and achievement. En R. Schulze y R.D. Roberts, International Handbook of Emotional Intelligence (pp.233-253). Cambridge: Hogrefe y Huber. Recuperado de https://bit.ly/2Pp2vgt

Graczyk, P.A., Weissberg, R.P., Payton J.W., Elias, M.J., Greenberg, M.T. y Zins, J.E. (2000). Criteria for evaluating the Quality of School-Based Social and Emotional Learning Programs. En R. Bar-On y J.D.A. Parker (Ed.), The Handbook of Emotional Intelligence: Theory, Development, Assessment and Application at Home, School, and in the Workplace (pp.391-410). San Francisco: Jossey-Bass.

Hernández Sampieri, R., Fernández Collado, C. y Baptista Lucio, P. (2014). Metodología de la investigación ( $6^{\mathrm{a}} \mathrm{ed}$.). México:McGraw Hill Education.

Hernández, M. (2015). La muestra en la investigación cualitativa. En N. Caparrós y E. Raya, Métodos y técnicas de investigación en trabajo social (pp.77-94). Madrid: Grupo 5.

Kemple, Kristen \& Lee, Ilrang \& Ellis, Stacy. (2019). The Impact of a Primary Prevention Program on Preschool Children's Social-Emotional Competence. Early Childhood Education Journal. 47(6), 641-652. doi:10.1007/s10643-019-00963-3

Kress, J. S. y Elias, M. J. (2007). School-Based Social and Emotional Learning Programs. In W. Damon, R. M. Lerner, K. A. Renninger y I. E. Siegel. Handbook of Child Psychology (pp. 592-618). Hoboken, NJ, US: John Wiley y Sons Inc. doi:10.1002/9780470147658.chpsy0415

León, O.G. y Montero, I. (2015). Métodos de investigación en Psicología y Educación. Las tradiciones cuantitativa y cualitativa. Madrid: MC Graw Hill.

Martinsone, B. (2016). Social Emotional Learning: Implementation of Sustainability-Oriented Program in Latvia. Journal of Teacher Education for Sustainability, 18 (1), 57-68. doi:10.1515/jtes-2016-0005

McCabe, P. C., y Altamura, M. (2011). Empirically valid strategies to improve social and emotional competence of preschool children. Psychology in the Schools, 48 (5), 513540. doi:10.1002/pits. 20570

O’Connor, C. A., Dyson, J., Cowdell, F., y Watson, R. (2017). Do universal school-based mental health promotion programmes improve the mental health and emotional wellbeing of young people? A literature review. Journal of Clinical Nursing, 27 (3-4), 412-426. doi:10.1111/jocn.14078

Pahl, K. M., y Barrett, P. M. (2007). The Development of Social-Emotional Competence in Preschool-Aged Children: An Introduction to the Fun FRIENDS Program. Australian Journal of Guidance and Counselling, 17 (1), 81-90. doi:10.1375/ajgc.17.1.81

Pérez, R. (2006). Evaluación de programas educativos. Madrid: La Muralla. 
Pérez-Escoda, N., Filella, G., Alegre, A., y Bisquerra, R. (2012). Desarrollo de la competencia emocional de maestros y alumnos en contextos escolares. Electronic Journal of Research in Education Psychology, 10 (28). doi:10.25115/ejrep.v10i28.1530

Quecedo, R. y Castaño, C. (2002). Introducción a la metodología de investigación cualitativa. Revista de Psicodidáctica, 14, 5-40. Recuperado de https://bit.ly/38Asb0V

Rodríguez, G., Gil, J. y García, E. (1996). Metodología de la investigación cualitativa. Málaga: Aljibe S.L.

Romero, S.J. y Ordóñez, X.G. (2018). Métodos, diseños y técnicas de investigación psicológica y educativa. Madrid: Udima.

Sabey, C. V., Charlton, C. T., Pyle, D., Lignugaris-Kraft, B. y Ross, S. W. (2017). A Review of Classwide or Universal Social, Emotional, Behavioral Programs for Students in Kindergarten. Review of Educational Research, 87 (3), 512-543. doi:10.3102/0034654316689307

Schindler, H. S., Kholoptseva, J., Oh, S. S., Yoshikawa, H., Duncan, G. J., Magnuson, K. A., y Shonkoff, J. P. (2015). Maximizing the potential of early childhood education to prevent externalizing behavior problems: A meta-analysis. Journal of School Psychology, 53 (3), 243-263. doi: 10.1016/j.jsp.2015.04.001

Stake, R. (2010). Investigación con estudio de Casos (5ª ed.).Madrid:Morata.

Stefan, C.A. (2018). Social-Emotional Prevention Programs for Preschool Children's Behavior Problems. doi.org/10.1007/978-3-319-74751-4

Yin, R. (2009). Case study research: Design and methods (4th Ed.). Thousand Oaks, CA: Sage.

Zeidner, M., Roberts, R.D. y Matthews, G. (2008). The science of Emotional Intelligence. European Psychologist, 13 (1), 64-78. doi: 10.1027/1016-9040.13.1.64 
